\title{
STUDIES OF THE BEAM-BEAM INTERACTION FOR THE LHC ${ }^{\#}$
}

\author{
$\underline{\text { S. Krishnagopal }}{ }^{*}$, Centre for Advanced Technology, Indore, INDIA \\ M. A. Furman and W. C. Turner, LBNL, Berkeley, CA, USA
}

\begin{abstract}
We have used the beam-beam simulation code CBI to study the beam-beam interaction for the LHC. We find that for nominal LHC parameters, and assuming only one bunch per beam, there are no collective (coherent) beambeam instabilities. We have investigated the effect of sweeping one of the beams around the other (a procedure that could be used as a diagnostic for head-on beam-beam collisions). We find that this does not cause any problems at the nominal current, though at higher currents there can be beam blow-up and collective beam motion.
\end{abstract}

\section{THE CODE CBI}

The code CBI (for Collective Beam-beam Interactions) is a self-consistent code that models the transverse beam-beam dynamics of beams of arbitrary distribution and ellipticity. It is a Particle-in-Cell (PIC) code that calculates the beambeam force on a two-dimensional (transverse) Cartesian grid. The code is evolving and presently has the following features:

(a) there is only one bunch per beam and there is only one collision point;

(b) the beams are ultra-relativistic;

(c) longitudinal dynamics is not modeled;

(d) arc transport is linear;

(e) radiation damping and fluctuations are put in once a turn and at one point in the ring;

(f) there is no crossing angle;

(g) transverse dimensions and distributions of the beams can be completely arbitrary.

Details of the code can be found in Refs. 1 and 2.

The code, as described above, is a strong-strong beambeam code that is best suited for studying collective beambeam effects in storage-ring $\mathrm{e}^{+} \mathrm{e}^{-}$colliders, particularly quadrupole effects (that affect the beam sizes). To our knowledge, quadrupole collective effects have never been studied for hadron colliders, and it seemed interesting and relevant to undertake this study for the Large Hadron Collider (LHC). In particular, in light of a proposal for sweeping one beam around the other as a diagnostic for head-on collisions [3], it seems relevant to look at possible beam-size blow-up and distortion as a consequence of quadrupole collective effects.

\section{RESULTS}

\subsection{Nominal LHC Parameters}

LHC parameters used in the simulations are given below in Table 1.

Table 1: Parameters for the LHC simulations.

\begin{tabular}{|l|c|}
\hline \multicolumn{1}{|c|}{ Parameter } & Value \\
\hline Energy $(\mathrm{TeV})$ & 7.0 \\
\hline Revolution period $(\mu \mathrm{s})$ & 88.9 \\
\hline Emittance $(\mathrm{nm}-\mathrm{rad})$ & 0.5 \\
\hline Beta function $(\mathrm{m})$ & 0.5 \\
\hline Tunes $(\mathrm{H}, \mathrm{V})$ & $(0.28,0.31)$ \\
\hline Nominal bunch current $(\mathrm{mA})$ & 0.2 \\
\hline Number of bunches & 1 \\
\hline
\end{tabular}

As can be seen from Table 1, the simulations use the nominal LHC parameters [4], except for the fact that we assume one bunch per beam, and therefore do not model parasitic beam-beam collisions. This is a feature we hope to incorporate into the code in the future.

We first ran our simulation for nominal LHC parameters, with the nominal bunch current of $0.2 \mathrm{~mA}$, and with an idealized feedback system that takes out all collective dipole effects (centroid motion). For reasons of computer time, the simulations were run for only 90,000 turns. Figure 1a shows the rms beam sizes for the last 20,000 turns of the simulation: it is clear that for nominal LHC parameters there are no collective quadrupole effects that could affect the performance. Beam sizes are pretty much equal to their nominal value, and there is no sizable beam blow-up. When the current is increased to $1 \mathrm{~mA}$ (Fig. 1b) there is some beam blow-up, but all beam sizes are the same, and there is no indication of collective behavior.

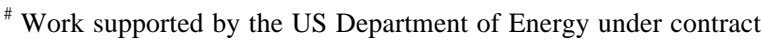
No. DE-AC-03-76SF00098. Presented at the PAC99, New York City, March 29-April 2nd, 1999.

*Email: skrishna@cat.ernet.in
} 

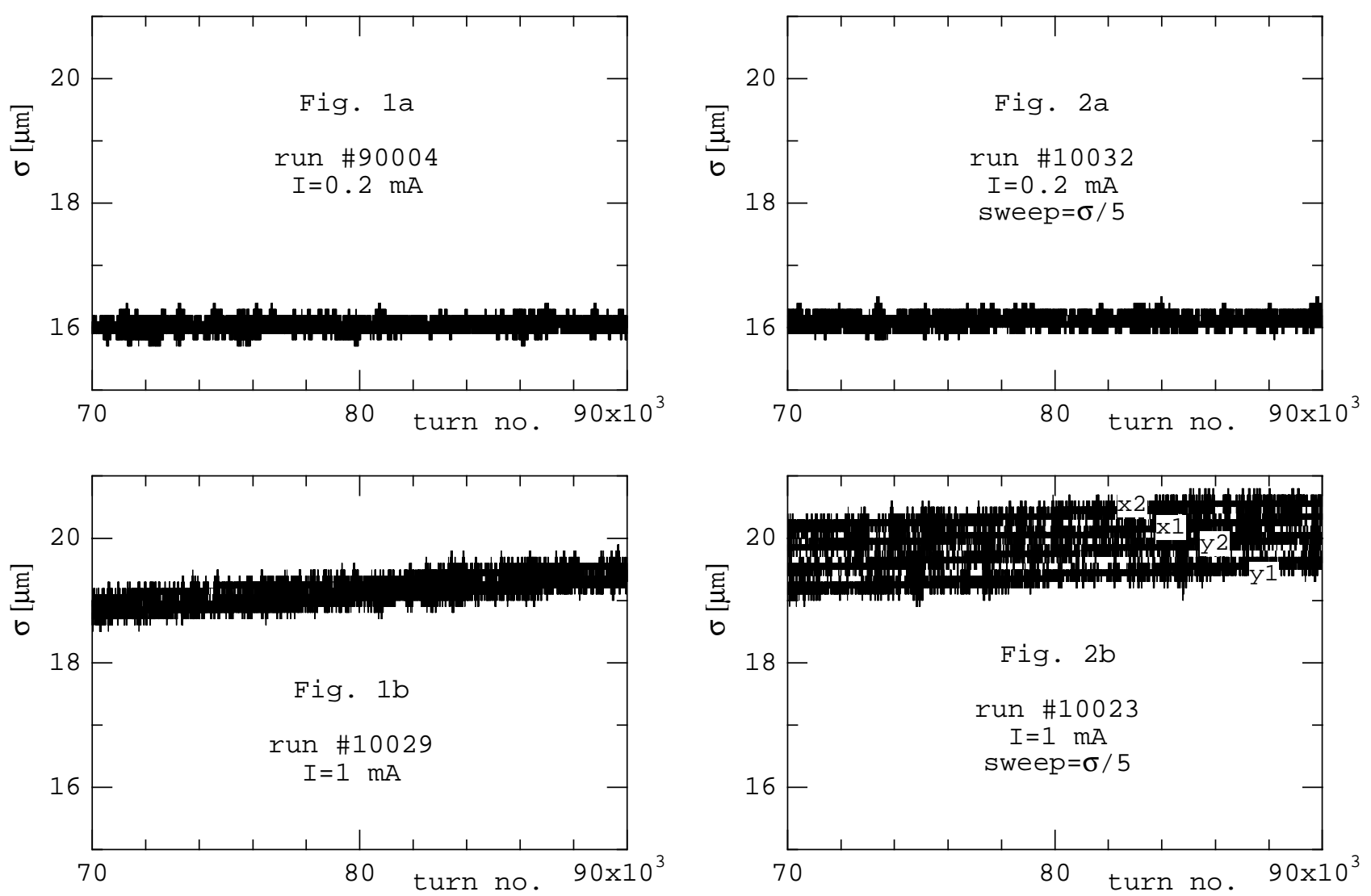

Figure 1: For nominal LHC parameters, plot of beam sizes as a function of turn number for the last 20,000 turns; (a) for the nominal current of $0.2 \mathrm{~mA}$, and (b) for a current of $1 \mathrm{~mA}$.

\subsection{Sweeping One Beam Around The Other}

We next looked at the effect of sweeping one beam around the other. We chose to sweep beam 1 around beam 2 . In this case, after each turn, as before, the centroids of the two beams are zeroed, implementing the idealized feedback system. Then the centroid of beam 1 is displaced a fixed radial distance from the zero position. Two input parameters govern this displacement: the displacement and the rotation period (in number of turns). The latter is the number of turns taken to sweep beam 1 once around beam 2 .

In the simulations described here, we fixed the rotation period at 10 turns, and looked at the effect on the beam sizes of different displacements and different currents. We looked at two different displacements, $\sigma / 5$ and $\sigma / 10$, where $\sigma$ is the nominal size of the beams $(15.8 \mu \mathrm{m})$. We looked at five different currents, starting from $0.1 \mathrm{~mA}$, up to 2 $\mathrm{mA}$. All simulations were run for 90,000 turns, and with the idealized feedback system turned on. Note that only one bunch is simulated in each beam: there are no parasitic collisions.

Figure 2: Beam size as a function of turn number for beam 1 being swept around beam 2 with a radial displacement of $\sigma / 5$; (a) at $I=0.2 \mathrm{~mA}$, and (b) at $\mathrm{I}=1$ $\mathrm{mA}$.

Figure 2 shows the beam-sizes as a function of turn number, for the last 20,000 turns, when the displacement is $\sigma / 5$. One can see that for the nominal current of 0.2 $\mathrm{mA}$ the beam-sizes are all equal, and equal to the nominal size of $15.8 \mu \mathrm{m}$, indicating that there are no deleterious collective beam-beam effects. At a current of $1 \mathrm{~mA}$, however, it is clear that the beams are being blown-up, and by unequal amounts: in other words, the beams are becoming elliptical, and there is a flip-flop developing. This is a signature of quadrupole collective beam-beam effects. The same picture is seen in Figure 3, for a displacement of $\sigma / 10$. Again, at a current of $0.2 \mathrm{~mA}$ the picture is benign, but at $1 \mathrm{~mA}$ a flip-flop has clearly set in.

Table 2 below gives a clearer picture of how the dynamics evolve with current. At a current of $0.1 \mathrm{~mA}$, there is no discernible blow-up of the beam, and no collective motion; all beam sizes are equal, and equal to the nominal size of $15.8 \mu \mathrm{m}$. When the bunch current is increased to the nominal $\mathrm{LHC}$ value of $0.2 \mathrm{~mA}$, there is slight beam blow-up, but all beam sizes are still equal, which indicates there is no collective motion. At a current of $0.5 \mathrm{~mA}$ there are the first signs of collective motion. All beam sizes are no longer equal: a flip-flop instability 
has developed. As the current is increased further, the beam blow-up as well as the flip-flop become larger.
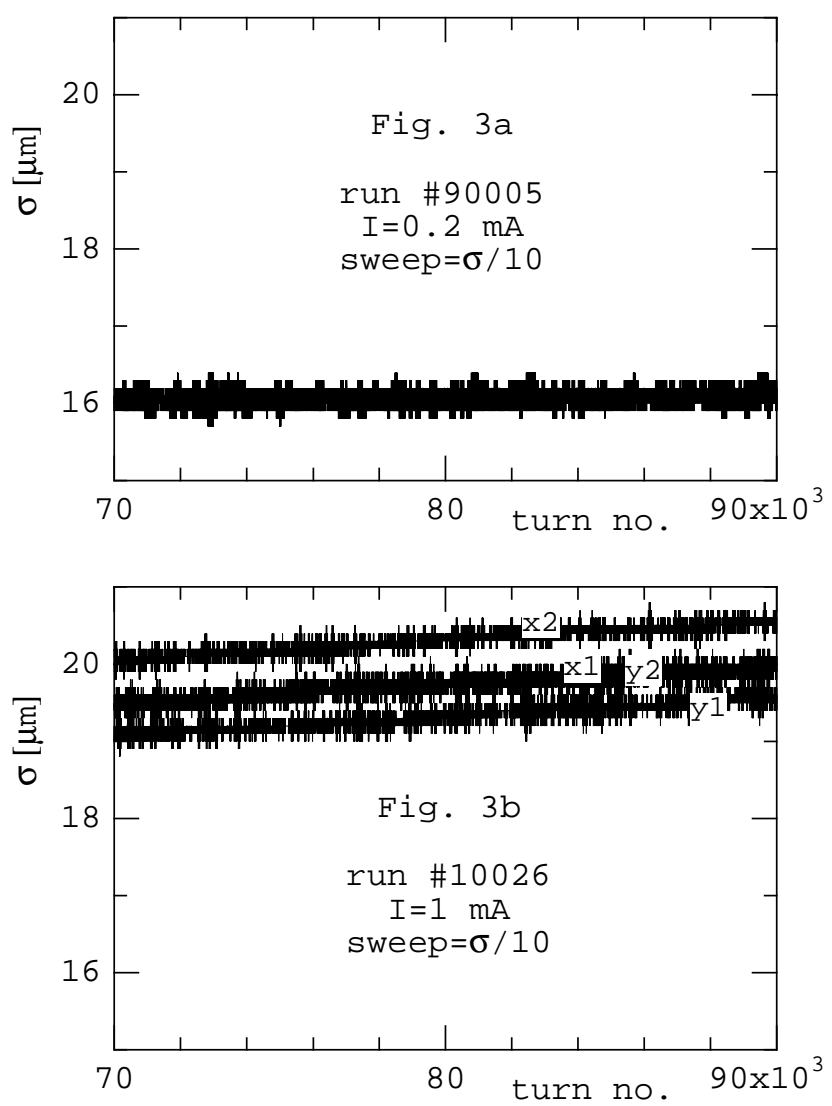

Figure 3: Beam size as a function of turn number for beam 1 being swept around beam 2 with a radial displacement of $\sigma / 10$; (a) at $\mathrm{I}=0.2 \mathrm{~mA}$, and (b) at $\mathrm{I}=1$ $\mathrm{mA}$.

Table 2: All beam sizes at the end of the simulation (after 90,000 turns), at different currents, for a displacement of $\sigma / 5$. Nominal beam size is $15.8 \mu \mathrm{m}$. Recall that beam 1 is being swept around beam 2 .

\begin{tabular}{|c|c|c|c|c|}
\hline $\begin{array}{c}\text { Current } \\
(\mathbf{m A})\end{array}$ & $\begin{array}{c}\sigma_{\mathbf{x} 1} \\
(\mu \mathbf{m})\end{array}$ & $\begin{array}{c}\sigma_{\mathbf{y} 1} \\
(\mu \mathbf{m})\end{array}$ & $\begin{array}{c}\sigma_{\mathrm{x} 2} \\
(\mu \mathbf{m})\end{array}$ & $\begin{array}{c}\sigma_{\mathbf{y} 2} \\
(\mu \mathbf{m})\end{array}$ \\
\hline $\mathbf{0 . 1}$ & 15.8 & 15.8 & 15.8 & 15.8 \\
\hline $\mathbf{0 . 2}$ & 16.2 & 16.1 & 16.1 & 16.1 \\
\hline $\mathbf{0 . 5}$ & 17.4 & 17.4 & 17.1 & 16.9 \\
\hline $\mathbf{1 . 0}$ & 20.6 & 20.0 & 20.4 & 19.6 \\
\hline $\mathbf{2 . 0}$ & 28.1 & 25.4 & 27.6 & 25.1 \\
\hline
\end{tabular}

\section{DISCUSSION AND CONCLUSIONS}

It is clear from Figs. (1a), (2a) and (3a), that for nominal LHC parameters, particularly the nominal bunch current of $0.2 \mathrm{~mA}$, there is little beam blow-up, and no collective motion, whether or not one beam is swept around the other. When one beam is swept around the other, collective effects are seen from a bunch current of around
$0.5 \mathrm{~mA}$ (over twice the nominal value), though at this current they are still small.

It should be emphasized that our simulations do not model multiple bunches and therefore parasitic collisions. These could have a significant impact on collective beambeam dynamics. We plan to extend the code to handle these effects.

In the simulations reported here dipole motion is removed by modeling an ideal feedback system in which the centroids of the two beams are zeroed every turn. The consequences of turning off this feedback, particularly on collective dipole motion, need to be explored. We plan to do this in the near future.

In conclusion, we have studied the beam-beam interaction at the LHC using the code CBI. We find that for nominal LHC parameters, collective quadrupole effects should not be an issue. If one beam is swept around the other, for diagnostic purposes, then collective issues still are unimportant at the nominal bunch current of $0.2 \mathrm{~mA}$, though they could become important at currents around and above twice the nominal value.

One of us (SK) would like to thank the other two for an invitation to visit the Lawrence Berkeley National Laboratory during the summer of 1998, when most of this work was done. We are grateful to NERSC for supercomputer support.

\section{REFERENCES}

[1] S. Krishnagopal, Phys. Rev. Lett. 76, 235 (1996).

[2] S. Krishnagopal, Centre for Advanced Technology Internal Report, CAT-95/5 (1995).

[3] Luminosity Instrumentation for the Absorbers in the Low Beta Insertions of the LHC, W. C. Turner, LBNL-42180(Aug. 1998), submitted for publication to PRST-AB.

[4] The Large Hadron Collider: Conceptual Design, CERN/AC/95-05 (LHC), 20 October 1995. 